\title{
Research on Distinguishing Technology of Frequency Hopping Network Signal
}

\author{
Yuan Yuan \\ School of Electronic and Information \\ Engineering \\ Beihang University \\ Beijing, China \\ Luna_369@163.com
}

\author{
Zhiyong Shi \\ College of Communication \\ Engineering, \\ Chongqing University \\ Chongqing, China \\ szy13@163.com
}

\author{
Jianguo Shen \\ ChongQing Communicate Institute \\ of PLA \\ ChongQing, China \\ cqshenjg@163.com
}

\begin{abstract}
The technology of extracting network information parameters from FH Synchronization Orthogonal network signal distinguishing is proposed in this paper, then its performance is simulated.
\end{abstract}

Keywords-FH Communication; Synchronization Orthogonal Network; Network Distinguish; Network Information Parameters

\section{INTRODUCTION}

Frequency hopping communication has strong capacity of resisting disturbance, good LPI characteristics as well as strong networking capability, which has been widely used in modern military communication. Therefore, to actively and effectively carry out research on frequency hopping communication reconnaissance is imperative now. At present, both domestic and overseas countries have made great progress on the aspect of frequency hopping signal reconnaissance, for instance, distinguishing issues of medium and low speed frequency hopping signal reconnaissance and non-orthogonal network signal have been resolved in a certain degree $^{[1]}$. However, there are still many problems of separating frequency hopping orthogonal network signal need to be further studied. Due to the application of frequency hopping network and battlefield integrated frequency hopping parameter management system, the characteristics of space frequency hopping signal become more and more complicated and elusive, which strengthened its LPI performance and increased the difficulty of net sorting. Based on this, some researches on distinguishing technology of frequency hopping network signal will be discussed in this paper.

\section{DEVELOPMENT STATUS OF DISTINGUISHING TECHNOLOGY OF FREQUENCY HOPPING SIGNAL RECONNAISSANCE}

To distinguish frequency hopping network signal, it is necessary to conduct reconnaissance of frequency hopping signal at first. There are many researches done for frequency hopping signal in abroad, but there's a few report about such aspect due to variety of difficulty or confidentiality. In fact, some certain researches have also been made in China, which put forward several methods on reconnaissance of frequency hopping signal, such as, adaptive and multi channel detection technology [1], compressed receiver detection technique ${ }^{[2]}$, digital channelization technology ${ }^{[3]}$, detection technology combined with compression and channelization ${ }^{[4]}$, maximum related treatment ${ }^{[5]}$, detection technology under sophisticated electromagnetic environment ${ }^{[6]}$ and so on. Generally speaking, the domestic reconnaissance of frequency hopping signal has a certain research foundation.

The traditional distinguishing technology focuses on completing distinguishing by extracting parameters from signal frequency, direction, amplitude and other characteristics of radio frequency or subtle features of signal ${ }^{[7.8 .9]}$. However, some of those parameters are not reliable due to impact of environment, some of them are difficult to be extracted, which result in several limits to these algorithms and concepts. Based on the literature of [10], this paper puts forward an algorithm about frequency hopping synchronous orthogonal network signal distinguishing from the prospective of combining reconnaissance and counter reconnaissance with a starting point of using network information parameter of frequency hopping signal into the distinguishing of synchronous orthogonal network, the key of implementation is how to extract network information parameters correctly and reliably.

\section{INTRODUCTION OF DISTINGUISHING ALGORITHM}

\section{A. Distinguishing model}

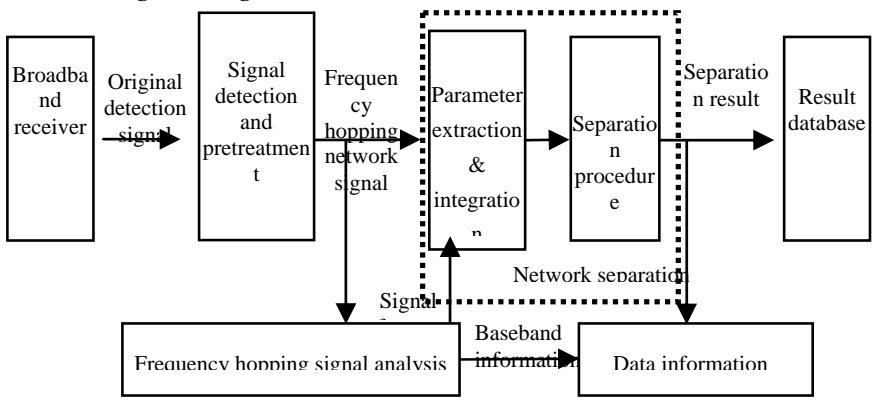

Fig.1. A distinguishing model of frequency hopping synchronous orthogonal network 
According to the distinguishing target that need to be achieved by synchronous orthogonal networking mechanism, signal characteristics and frequency hopping signal reconnaissance, the distinguishing model of frequency hopping synchronous orthogonal networking signal can be established as shown in Figure 1.

\section{B. Distinguishing algorithm}

In literature [5], the basic idea of distinguishing algorithm is that, distinguishing is achieved by extracting network information parameters carried by each network signal according to the different network information parameter based on different network information carried by all frequency hopping synchronous orthogonal networking signal. Signal with same network information belong to the same frequency hopping network, vice versa. The steps are as following:

(1) Parameter extraction and integration: Extract network distinguishing characteristic parameter based on analysis of basic feature of frequency hopping network signal, and then make classification, arrangement and integration, so as to form parameter database that can be directly called by distinguishing algorithm according to the needs of distinguishing.

(2) Distinguishing: mainly including two parts:

a)Conduct analysis, accumulation, extraction and integration of parameters by using several hops to generate distinguishing parameter set.

b)Conduct real-time distinguishing for several signal occurred simultaneously according to the gradual hop of distinguishing parameter set.

\section{EXTRACTION OF NETWORK INFORMATION PARAMETER}

The network information carried by each network is important parameter for the distinguishing of network. For typical frequency hopping synchronous orthogonal network, the network information is mainly including correlation code, network number and so on. Correlation code is a group of special code word used for synchronous maintenance and timing. Network number is the status symbol of each network used for network identification, while low speed correlation code is generally passed through during the usage, so that ensure the reliability of transmission. These two groups of parameter will be occurred in synchronous hop, data transfer, deferred-network-entry guiding hop and service hop signal when frequency hopping synchronous orthogonal network is working. One of typical frame structure in frequency hopping synchronous orthogonal network signal is shown in Table 1.

\section{TABLE I. EXTRACTION OF NETWORK INFORMATION PARAMETER}

\begin{tabular}{|c|c|c|c|c|c|}
\hline $\begin{array}{c}\text { Frequency } \\
\text { hopping }\end{array}$ & $\begin{array}{c}\text { Bit } \\
\text { synch } \\
\text { roniz } \\
\text { ation } \\
\text { Guid } \\
\text { ance }\end{array}$ & $\begin{array}{c}\text { Relevan } \\
\text { t code }\end{array}$ & $\begin{array}{c}\text { Network } \\
\text { number }\end{array}$ & $\begin{array}{c}\text { Synchronizatio } \\
\mathrm{n} / \text { Data/ } \\
\text { Deferred- } \\
\text { network-entry } \\
\text { guidance/Servi } \\
\text { ce information }\end{array}$ & $\begin{array}{c}\text { Prote } \\
\text { ction }\end{array}$ \\
\hline 116 & 100 & 24 & 64 & 768 & 80 \\
\hline
\end{tabular}

In order to correctly distinguish frequency hopping signal from different network by utilizing distinguishing algorithm, it is necessary to find the position of network information parameter contained in the signal of each jump at first, this is not only the primary issue of correct distinguishing of frequency hopping network, but also the key of distinguishing algorithm. The probability of correct extraction of network information parameter provided by such algorithm will directly influence the final probability of correct distinguishing, thus, the situation of such algorithm in this part will decide whether the whole distinguishing algorithm has superiority or not. This part is also the main content we need to research.

In order to extract this parameter, the position of first and final bit of correlation code and network number sequence shall be determined accurately. Specially for the frame structure of frequency hopping network shown in Table 1, the connection of correlation code and network number can be presented by one sequence, for example, $\mathrm{Ci}(\mathrm{i}=1,2,3 \ldots)$ indicates the correlation code and network number of No.i network. Regarding several frequency hopping signal captured at the same time, the correlation code and network number information can be extracted respectively through analysis and comparison of baseband information after the demodulation of several hop signal.

Supposing the speed, information speed, compression ratio and channel data speed of a frequency hopping signal is $250 \mathrm{H} / \mathrm{s}, 192 \mathrm{kbps}, 3: 2$ and $288 \mathrm{kbps}$ respectively, the bit distribution of its frame structure is shown as Table 1 . If there is no information send during frequency change, then 116 bit shall be set as " 0 ", bit synchronous guidance is the "1010..." code of 100 bit, and the correlation code, network code and code format are shown as Table 2. The synchronization/data/deferred-network-entry guidance/service information is 768 bit random $0 / 1$ sequence, while the protection bit is 80 bit random $0 / 1$ sequence.

TABLE II. FORMAT OF RELEVANT CODE, NETWORK NUMBER AND CODE

\begin{tabular}{|c|c|c|c|}
\hline & Relevant code & Network number & Code of network number \\
\hline Network-1 & 096E8F & 0001 & $0:$ F1EA; 1: 6F49; \\
\hline Network-2 & F69170 & 0010 & $0: 6$ F68; 1: 4583; \\
\hline Network-3 & F17690 & 0011 & $0:$ 52BD; 1: 8585; \\
\hline
\end{tabular}

Take three networks and three hops extraction as an example, the calculation of X11,X12,X13 with X21,X22,X23 shall be made respectively at first. As there is same frequency hopping, bit synchronous guidance, correlation code and network number in the information sequence of same network signal, the first 304 bit of same network signal information sequence after calculation without error are all zero, and the first 216 bit of different network signal information sequence after calculation are all zero, then, make calculation of X11,X12,X13 with X31,X32,X33 respectively. Similarly, the first 216 bit of different network signal information sequence after calculation are all zero, and the first 304 bit of same network signal information sequence after calculation without error are all zero. Due to the influence of data in the front and back end, redundant bit will be inevitably introduced accordingly. Assuming the probability of 0 and 1 occurring in the bit synchronous guidance sequence of front end and 
random sequence of back end is same, the probability of judging one more bit is $2(1 / 2) 3=1 / 4$. Take the smallest bit from $\mathrm{Ci}$ sequence of three networks as the bit of such sequence, then the probability of judging more bit of $\mathrm{Ci}$ sequence from three networks is $(1 / 4) 3=1 / 64$.

Figure 2(obtained when the error rate is 10-2) provide the calculation result (first 400 bit) of a certain signal sequence $\mathrm{X} 11$ of the first hop and six information sequences (X21,X22,X23,X31,X32,X33) within second and third hop of targeted network signal. From the figure, we can see that, the information sequence X11,X21,X31 of first and fourth column data obtained belong to the same frequency hopping network.

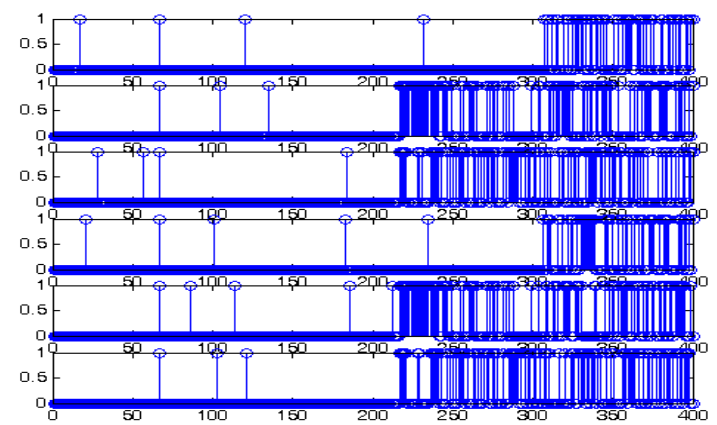

Fig.2. The calculation result (first 400 bit) of a certain signal sequence of first hop and six information sequences (X21,X22,X23,X31,X32,X33) within second and third hop of targeted network signal.

From the above process, we can find the error probability is relatively big when extract by three hops. If extract from four hops, similarly, the probability of judging one more bit is $2(1 / 2) 4=1 / 8$. Take the smallest bit from Ci sequence of three networks as the bit of such sequence, the probability of judging one more bit is (1/8)4=1/4096.

Thus, the error probability of extraction by four hops can be accepted basically. Considering the error code existed in channel and majority of judgment shall be required for modifying network information parameter, five hops signal will be used here for extracting network information. The steps are as following:

step1: Take 3 signals from the first hop for calculating with 12 signals within second, third, fourth and fifth hops respectively to obtain 36 groups of sequences.

step2: Find the first position pi that is not 0 in each group from 36 sequences, then arrange them in ascending order to get array $\mathrm{P}$.

step3: Take the first 24 numbers, using a matrix $q$ to arrange those 24 numbers from small to large to form a group to place into a certain line of the matrix. The column number will be depended by how many successive arrays, the number of matrix column will be depended on the number of such successive arrays. All columns will be compensate by " 0 ”.

step4: Take the line with most " 0 " from matrix, remove the largest and smallest value and take average value, i.e. the position number of first bit of network information parameter Ci. step5: Take last 12 values from $\mathrm{P}$, use matrix u to make continuous numbers from those 12 values into a group, and then put it into a certain line of matrix. The number of lines in matrix is equal to that of continuous array, compensate all lines with " 0 ”.

step6: Take the line with most " 0 " from matrix, remove the largest and smallest value and take average value, i.e. the position number of last bit of network information parameter Ci.

step7: Find the position of network information parameter $\mathrm{Ci}$ in the signal of each hop according to the first and last bit of network information parameter $\mathrm{Ci}$

step8: Take network information parameter Ci from 12 signals in second, third, fourth and firth hop, and then compare it with network information parameter $\mathrm{Ci}$ of 3 signals within first hop, if the same bit beyond a certain limit, those signals belong to same network.

step9: Compare the network information parameter Ci of 5 hops signal in same network, make majority decision for values in same position to obtain the modified network information parameter $\mathrm{Ci}$ of each network and store them.

\section{AlgORITHM SIMULATION}

Under MATLAB environment, we have made simulation of extracting network information parameter of 3 frequency hopping synchronous orthogonal network signals by using above method. All the parameters in simulation are assumed as Section 3. Please find the probability of correct extraction of network information parameter from a certain network signal under the condition of different error rate.

From Figure 3, we can see that, extraction of network information parameter of target network signal from several frequency hopping synchronous orthogonal networks can be achieved by using such algorithm through a greater probability under the condition of worse error respectively. However, the probability of correct extraction will be decreased with the increase of error rate. The probability of correct extraction is higher than $93 \%$ if the error rate is less than $1 \times 10^{-2}$, but it will starts to get worse when the error rate is more than $1 \times 10^{-2}$.

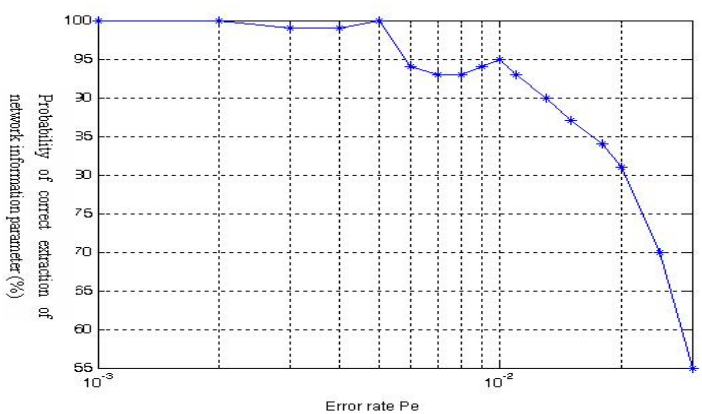

Fig.3. Probability of correct extraction of network information parameter of certain specified network signal under the condition of different error rate

\section{CONCLUSION}

This paper mainly researches the extraction issues of network information parameter by focusing on frequency 
hopping synchronous orthogonal network distinguishing algorithm, and provides a kind of algorithm of extracting network information parameter consequently. After verified by simulation, this algorithm is able to extract network information parameter of target network within a certain range of error rate under bad channel, which can supply firm foundation for the distinguishing of frequency hopping synchronous orthogonal network signal. However, such algorithm is only suitable for frequency hopping synchronous orthogonal network, there are still some limits for the application in frequency hopping asynchronous orthogonal network. Thus, it is necessary to further study another distinguishing algorithm for frequency hopping asynchronous orthogonal network.

\section{References}

[1] Ping Liangzi, Development Status of Hopping Frequency Signal Capture and Measurement Device in Foreign Countries [J], Foreign Military Electronic Warfare, No.4, 2003.

[2] Luo Laiyuan, Detection Technology of Spread Spectrum Low Intercept Rate Signal[J], Telecommunication technology research, No.10 , 1997.
[3] Wang Jun, Application of Digital Channel Technology in Frequency Hopping Signal Reconnaissance[J], Telecommunication Technology Research, 2000.

[4] Feng Fuqiang, Liu Deshu, A New Method for Capturing, Classification and Identification of Frequency Hopping Signal[J], Journal of the Chinese PLA Electronic Engineering Institute, No.4, 1995.

[5] Xu Qihua, The Application of Maximum Relative Treatment in Ultrashort Wave Frequency Hopping Signal Reconnaissance[j], Telecommunication Technology Study, No.1 , 2000.

[6] Yang Hongwa, Detection Technology of Frequency Hopping Signal Under Sophisticated Electromagnetic Environment [C], Proceedings of 12th Academic Annual Meeting of Electronic Countermeasures Branch of Chinese Institute of Electronics.

[7] Wrote by James Tsui, Translated by Yang Xiaoniu, Lu Annan and Jin Biao, Broadband Digital Receiver[M], Beijing: Electronic Industry Press, 2002. 10.

[8] Yao Fuqiang, Military Telecommunication Anti-interference and Network Application[M], Beijing: PLA Press, 2004. 4.

[9] Wang Mingsan etc. Telecommunication Countermeasure Principle[M], Beijing: PLA Press, 1999.11.

Wu Fan, Li Yusheng, Yao Fuqiang, A Seperation Algorithm of Frequency Hopping Synchronous Orthogonal Network Signal[C], Proceedings of 19th Graduate Students Telecommunication Meeting in Nanjing, 2004.11. 